\title{
Las colecciones biológicas: Los tesoros escondidos de un país mega-diverso.
}

\author{
Claudia Segovia-Salcedo ${ }^{1,2}$, Luis Carrasco ${ }^{3}$ y Néstor Acosta Buenaño ${ }^{3}$ \\ ${ }^{1}$ Universidad de las Fuerzas Armadas, Departamento de Ciencias de la Vida, Sangolquí, Ecuador.. \\ mcsegovia@espe.edu.ec \\ ${ }^{2}$ Proyecto iDigBio. University of Florida, Florida Museum of Natural History, Gainesville, Fl. USA. \\ ${ }^{3}$ Ministerio del Ambiente. Subsecretaría de Patrimonio Natural del Ecuador. Quito, Ecuador \\ luis.carrasco@ambiente.gob.ec, nestor.acosta@ambiente.gob.ec
}

Recibido: 2015-07-31; aceptado: 2015-10-05

Ecuador, a pesar de su reducido tamaño, alberga una de las más altas concentraciones de diversidad en el planeta y mucho de ella no está reflejada en nuestras colecciones biológicas (Suárez, 2014). Su ubicación en la intersección de la línea ecuatorial, junto con los Andes y la Amazonía, contribuye a su gran riqueza biológica. Grandes naturalistas como el Baron Alexander von Humboldt (18021803), Jacques-Alexandre Goujoud Bonpland (1799-1805), Charles Darwin (1835) entre otros, han explorado y colectado en el Ecuador, y muchos de esos especímenes ecuatorianos se encuentran en los museos de Historia Natural más importantes del mundo sin que científicos locales puedan acceder. En la actualidad, la mayoría de los especímenes ecuatorianos de colecciones recientes se encuentran en instituciones locales públicas y privadas, sin embargo, no sabemos con exactitud el número de colecciones y de especímenes debido a la falta de integración y apertura a la socialización de quienes poseen estos datos. Esto ocasiona que esta importante información no esté de forma organizada y al alcance de investigadores locales e internacionales, tomadores de decisiones y público en general. A pesar que a nivel mundial se están creando bases de datos y portales de biodiversidad con acceso libre como Global Biodiversity Information Facility (GBIF), Atlas of Living Australia (www.ala.org.au), Integrated Digitized Biocollection (www.idigbio.org), CONABIO (Comisión Nacional para el Conocimiento y uso de la Biodiversidad) (Janken, 2013), en nuestro país no hemos iniciado un proceso nacional de inventario de las colecciones biológicas para una posterior digitalización. Los procesos de democratización de la información de biodiversidad están creando una mayor interacción entre el público y las colecciones, fortaleciendo su existencia y además explorando nuevas aplicaciones para el uso de las mismas en diferentes sectores de la sociedad (Drew, 2011). El objetivo de este artículo es presentar los resultados de un breve inventario de las colecciones biológicas del Ecuador con miras a la creación de la Base Nacional de Datos de Biodiversidad (BNDB) y su portal web dentro de la Subsecretaría de Patrimonio Natural del Ecuador del Ministerio del Ambiente.

En los meses de enero a mayo del presente año se inició este proceso como un primer intento de integración de los herbarios, museos y colecciones biológicas del Ecuador. A través de encuestas y visitas a diferentes instituciones se obtuvo el número de especímenes, tipos y fecha de fundación. Si bien hemos logrado recopilar valiosa información, algunas colecciones todavía deber ser encontradas e incluidas. Como resultado de este proceso descubrimos verdaderos tesoros para la comunidad científica que se encuentran depositados en nuestro país. El Instituto de Ciencias Biológicas de la Escuela Politécnica Nacional (EPN) tiene la única colección paleontológica con más de 10000 especímenes: alberga las colecciones mastozoológica e ictiológica más grandes del Ecuador con ejemplares que datan de inicios del siglo anterior como un mono araña de la costa, Ateles fusciceps (1969, figura 1a), un mono capuchino, Cebus albifrons, (1925, figura 1b) que según Tirira (2015) actualmente corresponde a Cebus aequatorialis, y musaraña andina, Cryptotis equatoris (1951, figura 1c). Más de 30000 ejemplares de mamíferos se encuentran depositados en las colecciones de la Pontificia Universidad Católica del Ecuador (QCAZ), el Instituto de Ciencias Biológicas de la EPN y el Museo Ecuatoriano de Ciencias Naturales (MECN), con una representatividad taxonómica del $70 \%$ de nuestra mastofauna. 
En el caso de los peces, las colecciones ictiológicas de la EPN y el MECN albergan más de 10000 especímenes catalogados y muchos más por ser identificados. La mayoría de las muestras pertenecen a la región de la Amazonía y representan especialmente a las familias Characidae y Loricaridae, convirtiéndole en un centro de referencia para la zona norte de esta región.

Las colecciones entomológicas son las más grandes del país con más de 1'300000 ejemplares distribuidos en cinco museos: QCAZ, MECN, EPN, Biblioteca Aurelio Espinoza Pólit y USFQ. El museo QCAZ es el que alberga la mayor cantidad de especímenes con cerca de un millón de invertebrados, los grupos con mayor representatividad son los Carábidos y Lepidópteros. Otras colecciones importantes de insectos se encuentran en la Fundación Biblioteca Ecuatoriana Aurelio Espinoza Pólit (200 000 mariposas) y en la Universidad San Francisco de Quito con un enfoque en invertebrados acuáticos (aprox. 10 órdenes, número de especies no determinado). El Museo Ecuatoriano de Ciencias Naturales (MECN) es el mayor repositorio de pieles de aves en el Ecuador con más de 8000 especímenes con una representatividad del $80 \%$ de las especies de aves del país, que albergan varios tipos y especímenes únicos.

En laúltima década las colecciones herpetológicas han crecido exponencialmente con énfasis en los Andes y la Amazonía, alcanzando más de 40000 especímenes. Los esfuerzos de varias instituciones (QCAZ, JAMBATU, MECN) han llevado a un incremento en el número de descripciones de nuevas especies y por ende de publicaciones científicas (más de 30 artículos en una búsqueda utilizando las palabras claves Ecuador y Herpetología en los últimos 10 años a tráves de la base de datos Science Direct).

Finalmente, entre los 13 herbarios que brindaron información mantienen más de 650000 especímenes con énfasis en la Flora Amazónica y Andina con información desde mediados del siglo anterior. Las colecciones botánicas han permitido clarificar el número, rango de distribución, y estado de conservación de muchas de las poblaciones vegetales en nuestro territorio, así como la generación de varias obras de recopilación de la Flora del Ecuador. Sin duda los herbarios han sido las colecciones mejor organizadas y preservadas en nuestro país. Es interesante conocer
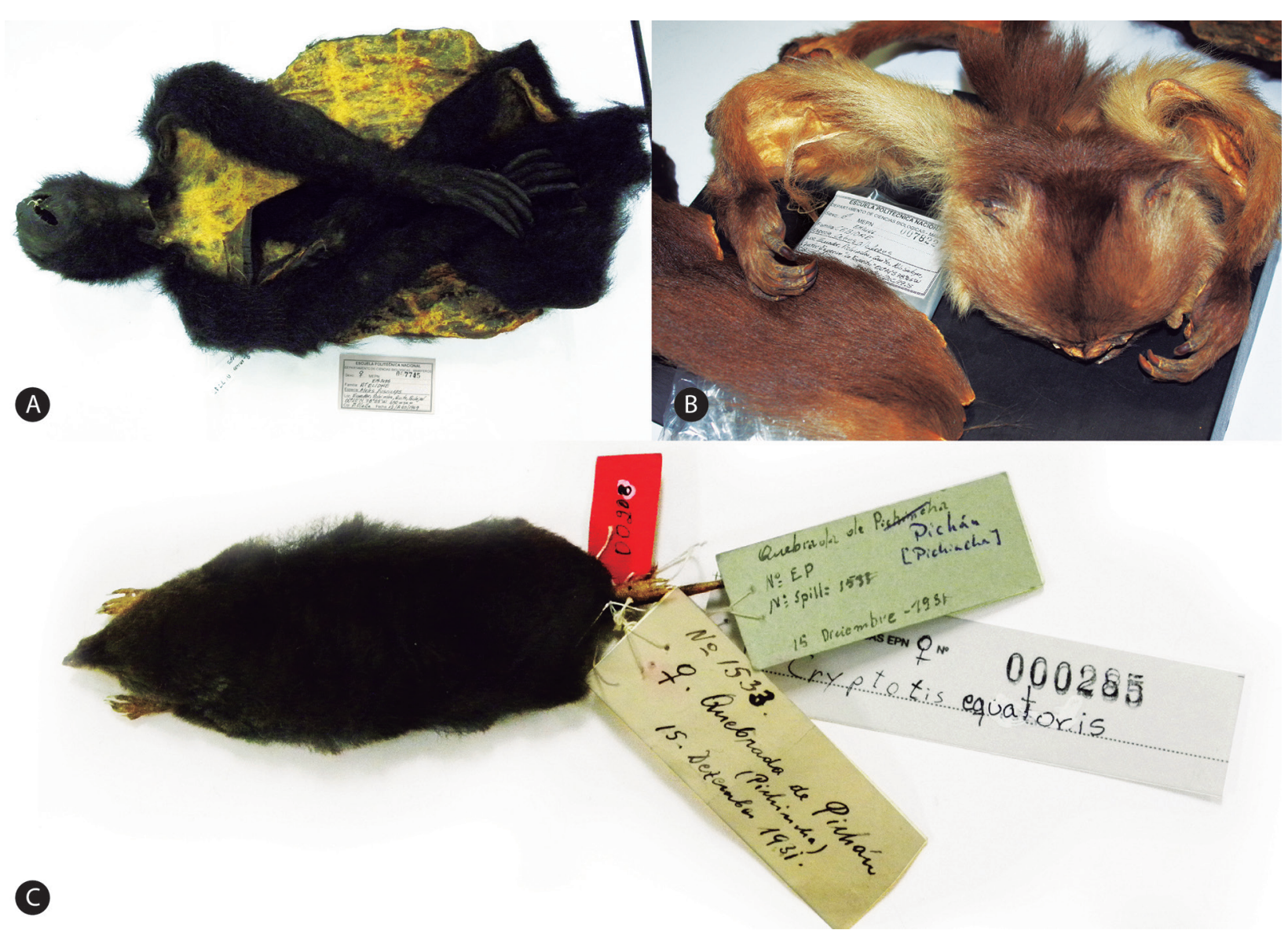

Figura 1. Los especímenes más antiguos encontrados en las colecciones biológicas ecuatorianas ubicados en el Instituto de Ciencias Biológicas de la Escuela Politécnica Nacional. A) Mono Araña de la Costa (Ateles fusciceps, 1969). B) Mono capuchino, Cebus albifrons, (1925). C) Musaraña Andina (Cryptotis equatoris, 1951). 
que en el Ecuador existe un herbario de Botánica Económica en la USFQ, una Xiloteca (Herbario de la Universidad Técnica del Norte) y una colección botánica histórica perteneciente al Padre Sodiro en la Fundación Biblioteca Ecuatoriana Aurelio Espinoza Pólit (Tabla 1, Figura 2).

Tabla 1. Datos recolectados de las colecciones biológicas ecuatorianas en esta investigación.

\begin{tabular}{|c|c|c|c|c|}
\hline \multicolumn{5}{|c|}{ HERBARIOS } \\
\hline Institución & Acrónimo & Año & Registros & Tipos \\
\hline Universidad Central del Ecuador & Q & 1860 & 12535 & \\
\hline Unidad Educativa Bolívar & UEB & 1921 & 3000 & \\
\hline Universidad Nacional de Loja & LOJA & 1949 & 45000 & 66 \\
\hline Escuela Superior Politécnica del Chimborazo & CHEP & 1966 & 16724 & \\
\hline Pontificia Universidad Católica del Ecuador & QCA & 1971 & 200000 & 1000 \\
\hline Museo Ecuatoriano de Ciencias Naturales & QCNE & 1977 & 238598 & 1888 \\
\hline Universidad Técnica del Norte & HUTN & 1985 & 15000 & \\
\hline Universidad Central del Ecuador & QAP & 1989 & 90000 & \\
\hline Universidad San Francisco de Quito & QUSF & 1994 & 29484 & 7 \\
\hline Universidad Técnica Particular de Loja & HUTPL & 2002 & 15000 & \\
\hline Universidad Técnica del Norte & HUNT & 2005 & 1800 & \\
\hline Universidad Tecnológica Indoamérica & HUTI & 2012 & 900 & 2 \\
\hline Fundacion B.E. Aurelio Espinoza Polit & QPLS & 2013 & 13500 & 219 \\
\hline Universidad Tecnica de Cotopaxi & UTC & 2015 & 3000 & \\
\hline TOTAL & & & 684541 & 3182 \\
\hline \multicolumn{5}{|c|}{ MUSEOS - VERTEBRADOS } \\
\hline Institución & Acrónimo & Año & Registros & Tipos \\
\hline Universidad Central del Ecuador & Q & 1860 & 1654 & \\
\hline Unidad Educativa Bolívar & UEB & 1921 & 3000 & \\
\hline Colegio Teodoro Gómez de la Torre & CTGT & 1944 & 160 & \\
\hline Escuela Politécnica Nacional & EPN & 1946 & 55259 & 159 \\
\hline Universidad Técnica de Ambato & MCCUTA & 1969 & 555 & \\
\hline Pontificia Universidad Católica del Ecuador & QCAZ & 1973 & 15500 & \\
\hline Escuela Superior Politécnica del Chimborazo & ESPOCH & 1975 & 20000 & \\
\hline Instituto Nacional de Biodiversidad - MECN & MECN & 1977 & 42023 & 52 \\
\hline FundaciónHerpetológica Gustavo Orcés & FHGO & 1989 & 12000 & \\
\hline Universidad Técnica Particular de Loja & MUTPL & 2005 & 500 & \\
\hline Centro - Jambatu & $\mathrm{CJ}$ & 2011 & 760 & 1 \\
\hline Pontificia Universidad Católica- Ambato & PUCA & 2011 & 339 & \\
\hline Universidad Tecnológica Indoamerica & MUZUTI & 2011 & 8186 & \\
\hline Universidad San francisco de Quito & MUSI & 2014 & 7600 & \\
\hline Fundacion B.E. Aurelio Espinoza Polit & FDPR & & 200000 & \\
\hline TOTAL & & & 367536 & 212 \\
\hline \multicolumn{5}{|c|}{ MUSEOS - INVERTEBRADOS } \\
\hline Institución & Acrónimo & Año & Registros & Tipos \\
\hline Escuela Politécnica Nacional & EPN & 1946 & 79357 & 42 \\
\hline Pontificia Universidad Católica del Ecuador & QCAZ & 1973 & $1^{\prime} 000000$ & 2683 \\
\hline Instituto Nacional de Biodiversidad - MECN & MECN & 1977 & 59227 & 71 \\
\hline Universidad Técnica del Norte & UTN-in & 2005 & 1800 & \\
\hline TOTAL & & & $1^{\prime} 140384$ & 2796 \\
\hline
\end{tabular}




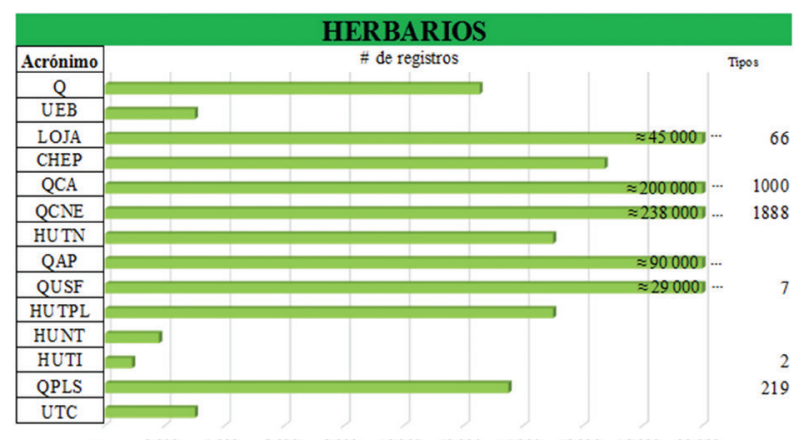

$\begin{array}{llllllllll}2,000 & 4,000 & 6,000 & 8,000 & 10,000 & 12,000 & 14,000 & 16,000 & 18,000 & 20,000\end{array}$
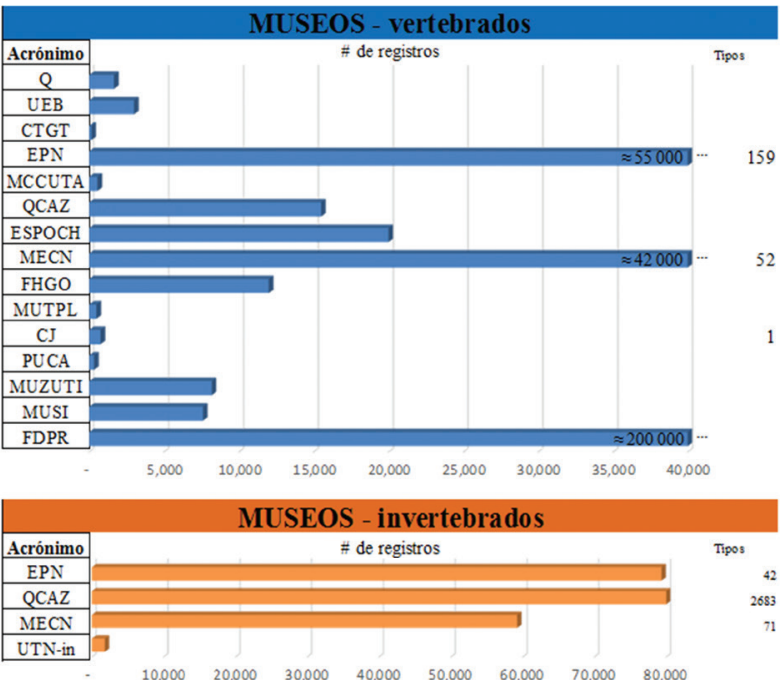

Figura 2. Número de registros y tipos reportados en las colecciones biológicas ecuatorianas

Las colecciones biológicas son verdaderos legados de información que necesitan ser apoyados, mantenidos y protegidos por el Estado e instituciones privadas (Hill et al., 2012; Stucky et al., 2014). No solamente son el sitio de referencia científica para el descubrimiento de nuevas especies, sino que son sitios de generación de conocimiento en diferentes ámbitos (Winker, 2004; Matsunanga et al., 2013). Una colección bien curada puede ser fundamental para la creación de modelos de cambio climático que incluyan rangos de expansión y reducción de distribución, variación fenológica o cambios evolutivos. Además, las colecciones biológicas permiten documentar cambios en la estructura de la comunidad en épocas recientes o pasadas (Drew, 2011; Graham et al., 2004; Lister \& Climate Change Research Group, 2011), de ahí la importancia de los repositorios históricos como las muestras del padre Sodiro que se encuentran albergadas en la Fundación Biblioteca Ecuatoriana Aurelio Espinoza Pólit, o las muestras paleontológicas del EPN. Mediante información de estas muestras como los rangos de distribución, podemos conocer los procesos de disminución poblacional y así determinar áreas prioritarias de conservación para especies endémicas o amenazadas (Fisher \& Davidson, 1998; Graham et al., 2004; O'Connell et al., 2004; Shaffer et al., 2009), además los datos de los herbarios nos ayudan a entender los procesos de introducción y distribución de especies invasoras. A través de la información depositada en las colecciones podemos determinar detalles de la historia natural de los organismos (Krishtalka y Humphrey 2000; Miller y Rogers, 2014). Los especímenes de museos nos permiten definir indicadores de impacto ambiental en los diferentes ecosistemas al ser fuente de material genético, bioquímico e isotópico, así como determinar vectores y controladores biológicos de enfermedades (Graham et al., 2004; Lister \& Climate Change Research Group, 2011).

En los últimos años, las colecciones han influenciado en la aplicación y el desarrollo de nuevas herramientas bioinformáticas para la digitalización e integración de bases de datos (Edwards et al., 2000; Berents et al., 2010; Drew, 2011). Finalmente, no podemos olvidar que las colecciones biológicas juegan un papel fundamental en la educación en todos los niveles, donde se puede incentivar procesos de asociación, observación y análisis de los objetos desde los niños hasta los adolescentes (Cook et al., 2014; Powers et al., 2014; Knight-Davis et al., 2015). Los especímenes de las colecciones son una herramienta clave en el entrenamiento de los estudiantes de pregrado, carreras biológicas y afines. Los museos de historia natural y las colecciones biológicas son uno de los pocos sitios donde convergen el público en general y los científicos creando el ambiente adecuado para entablar una comunicación directa sobre temas de conservación y manejo (Drew, 2011).

A pesar de la falta de apoyo por parte de las entidades estatales tanto a nivel locales como central (bajo presupuesto, falta de capacitación e infraestructuras adecuadas), las colecciones biológicas en nuestro país son numerosas y han permanecido activas por más de un siglo, pero la información todavía está incompleta, dispersa, y muchas veces inaccesible. Necesitamos entonces, crear una plataforma que nos permita compartir información para unir esfuerzos en áreas geográficas y taxonómicas de interés, evitar la duplicación de trabajo, y fortalecer a las colecciones pequeñas, de ahí la iniciativa de la creación de la Base Nacional de Datos de Biodiversidad (BNDB) con los objetivos de investigación, educación, manejo y conservación de nuestro recurso más preciado: la Biodiversidad. El conocimiento sobre nuestra biodiversidad está cambiando rápidamente y necesitamos generar mecanismos que nos permitan utilizar y contribuir a esa información con un manejo responsable y multidisciplinario de los datos. 
El valorar nuestras colecciones biológicas es un deber de todos los ciudadanos. Conocerlas, apoyarlas, difundirlas, y protegerlas es una misión no solo de la comunidad científica y quienes las posean, sino del Estado y de la sociedad. Esto se fundamenta en que las colecciones biológicas guardan nuestra herencia de conocimiento para las futuras generaciones. Una razón más por lo cual la colaboración entre los curadores y los investigadores es esencial para la inclusión de las colecciones en los proyectos de investigación. Es tiempo de crear una alianza nacional en favor de las colecciones biológicas para promover su continuidad en el tiempo y su apertura a los desafíos del siglo XXI a través de una colaboración entre academia y estado.

\section{AGRADECIMIENTOS}

Los autores agradecen a todas las instituciones que abrieron sus puertas y compartieron su información. A la Pontificia Universidad Católica del Ecuador (QCAZ-mastozoología, QCA, Ambato), Instituto de Ciencias Biológicas de la Escuela Politécnica Nacional, Universidad Central del Ecuador (Q y QAP), Instituto Nacional de Biodiversidad (MECNQCNE), al Herbario de la Universidad Nacional de Loja, Fundación Herpetológica Gustavo Orcés, Centro Jambatu de Investigación y Conservación de Anfibios; Universidad San Francisco de Quito (QUSF y MUSF), Universidad Técnica del Norte (HUTN), Universidad Tecnológica Indoamérica (HUTI), Universidad Técnica de Cotopaxi, Escuela Superior Politécnica del Chimborazo (Herbario y Museo), Unidad Educativa Bolívar, Universidad Técnica Particular de Loja (HUTPL), Fundación Biblioteca Ecuatoriana Aurelio Espinoza Polit, Colegio Teodoro Gómez de la Torre, Universidad Técnica de Ambato, y a Silva Carbón por su auspicio. Parte de la información presentada pertenece al proyecto del Ministerio del Ambiente "Inventario de Centros de Rescate".

\section{REFERENCIAS BIBLIOGRÁFICAS}

Berents P, Hamer M y Chavan V. 2010. Towards demand driven publishing: approaches to the prioritization of digitization of natural history collections data. Biodiversity Informatics, 7(2):113-119.

Cook JA, Edwards SV, Lacey EA, Guralnick RP, Soltis PS, Soltis DE y Ickert-Bond S. 2014. Natural History Collections as Emerging Resources for Innovative Education. BioScience, 64(8):725-734
Drew J.2011. The Role of Natural History Institutions and Bioinformatics in Conservation Biology. Conservation Biology, 25(6):1250-1252.

Edwards JL, Lane MA y Nielsen ES. 2000. Interoperability of biodiversity databases: biodiversity information on every desktop. Science, 289(5488), 2312-2314.

Graham CH. Ferrier S. Huettman F. Moritz C y Peterson AT.. 2004. New Developments in museum-based informatics and application in biodiversity analysis. Trends in Ecology and Evolution, 19(9):497-502.

Hill A, Gularnick R, Smith A, Sallans A, Gillespie $\mathrm{R}$, Denslow $\mathrm{M}$, et al. 2012. The notes from nature tool for unlocking biodiversity records from museum records through citizen science. ZooKeys, 209:219-233.

Janken J. 2013. Biodiversity Online: Toward a Network Integrated Biocollections Alliance. Bioscience, 63(10):789-790.

Knight-Davis S, Bruns T y Tucker G. 2015. Big Things Have Small Beginnings: Curating a Large Natural History Collection-Processes and Lessons Learned. Journal of Librarianship and Scholarly Communication, 3(2).

Krishtalka L y Humphrey PS. 2000. Can natural history museums capture the future? BioScience, 50(7):611-617.

Lister AM y Climate Change Research group. 2011. Natural History Collections as source of long term datasets. Trends in Ecology and Evolution, 26(4):153-154.

Matsunaga A, Thompson A, Figueiredo RJ, Germain-Aubrey CC, Collins M, Beaman RS y Fortes J. 2013. A computational-and storage-cloud for integration of biodiversity collections. En eScience (eScience), 2013 IEEE 9th International Conference on pp. 78-87.

Miller JT y Jolley-Rogers G. 2014. Correcting the disconnect between phylogenetics and biodiversity informatics. Zootaxa, 3754(2):195-200.

O'Connell AF, Gilbert AT y Hatfield JS. 2004. Contribution of natural history collection data to biodiversity assessment in national parks. Conservation biology, 18(5):1254-1261. 
Powers KE, Prather A, Cook J, Woolley J, Bart HL, Monfils AK y Sierwald P. 2014. Revolutionizing the Use of Natural History Collections in Education. Science Education Review, 13(2):24-33.

Shaffer HB, Fisher RN y Davidson C. 1998. The role of natural history collections in documenting species declines. Trends in Ecology and Evolution, 13(1):27-30.

Stucky BJ, Deck J, Conlin T, Ziemba L, Cellinese N y Guralnick R. 2014. The BiSciCol Triplifier: bringing biodiversity data to the Semantic Web. BMC bioinformatics, 15(1):257

Suárez L. 2014. ¿Cómo se explica tanta biodiversidad en el Ecuador? pp. 50-51 en: García, M., D. Parra P. y P. Mena V. 2013. El País de la Biodiversidad: Ecuador. Fundación Botánica de los Andes, Ministerio del Ambiente y Fundación EcoFondo. Quito.
Winker K. 2004. Natural history museums in a postbiodiversity era. BioScience, 54(5):455-459.

Tingley MW y Beissinger SR. 2009. Detecting range shifts from historical species occurrences: new perspectives on old data. Trends in Ecology and Evolution, 24(11):625-633.

Tirira D. 2015. Mamíferos del Ecuador: Lista Actualizada de Especies del Ecuador. 